\title{
Cure dialitiche domiciliari. Una frontiera da raggiungere, senza trascurare le criticità
}

\author{
Giuseppe Vanacore ${ }^{1}$, Antonio Santoro ${ }^{2}$ \\ ${ }_{1}^{1}$ Presidente ANED Associazione Nazionale Emodializzati Dialisi e Trapianto - ONLUS, Milano - Italia \\ ${ }^{2}$ Direttore Comitato Scientifico ANED Associazione Nazionale Emodializzati Dialisi e Trapianto - ONLUS, Milano - Italia
}

\begin{abstract}
Home dialysis treatments. A frontier to be reached, without ignoring critical issues
The National Plan of Chronicity, approved by the Italian State-Regions Conference, refers to the topic of home care, specifying how the fundamental objective of chronic care systems is to keep the sick person at home as much as possible. Currently, home dialysis - both peritoneal and haemodialysis - uses high-quality safe technology systems and allows patients to perform therapy safely at home. ANED wishes to stress that the choice of dialysis treatment, which certainly depends on clinical evaluations, must at the same time consider the psychological and emotional aspects of the patient, the expectations and needs of his life and the social context, aiming at an increasingly personalized and sustainable therapy for patients and the healthcare system.
\end{abstract}

Keywords: Chronic kidney disease, Home care, Home dialysis, Peritoneal dialysis

Nel periodo di lockdown imposto dalla pandemia COVID19 , alle oggettive difficoltà di contrastare la diffusione del contagio se ne sono aggiunte altre dovute alla tendenza della nostra Sanità di essere incentrata prevalentemente sull'attività ospedaliera a discapito della medicina territoriale e della prevenzione. Si è dovuto constatare che, dove le reti territoriali di cura e i trattamenti domiciliari sono andati in crisi, come è avvenuto in Lombardia, maggiori sono state le difficoltà di fare fronte al coronavirus.

D'altronde, da tempo, continue sollecitazioni, per reinvestire sulla prevenzione e sulla cronicità, provengono dal mondo scientifico, ma anche da significativi settori della politica e dalle associazioni, tra le quali sicuramente ANED. Vi è la consapevolezza che bisogna qualificare ed estendere le cure domiciliari. II Ministro Roberto Speranza ha prontamente recepito i vari input, sottolineando in diverse occasioni la necessità di aprire una fase nuova, garantendo le risorse necessarie per il Servizio Sanitario Nazionale, per sostenere in particolare un adeguato sistema di cure universalistiche in

Received: October 27, 2020

Accepted: October 28, 2020

Published online: December 7, 2020

Indirizzo per la corrispondenza:

Giuseppe Vanacore

Presidente ANED

Associazione Nazionale Emodializzati Dialisi e Trapianto - ONLUS

Via Ulrico Hoepli, 3

20121 Milano - Italia

presidente@aned-onlus.it modo uniforme sul territorio nazionale, senza trascurare la dignità del paziente e la sua qualità di vita.

Il punto di vista che ANED intende sottolineare, avendo come riferimento di base il Piano Nazionale delle Malattie Croniche (1) e in qualità di Associazione Nazionale di tutti i nefropatici, dializzati e trapiantati, riguarda i problemi che si accompagnano all'obiettivo della deospedalizzazione delle cure. A quest'ultima, infatti, occorre affiancare alcune proposte che devono accompagnare la devoluzione delle cure verso il territorio, al fine di una diffusione e di una condivisione maggiori dei pazienti e dei loro familiari.

II SSN ha, fra i suoi obiettivi, la deospedalizzazione per le cure dei malati cronici. II Piano Nazionale della Cronicità, approvato dalla Conferenza Stato-Regioni, pone come riferimento il tema delle cure domiciliari, specificando come l'obiettivo fondamentale dei sistemi di cura della cronicità sia quello di mantenere il più possibile la persona malata al proprio domicilio e di impedire o, comunque, ridurre, il rischio di istituzionalizzazione, senza far ricadere sulla famiglia tutto il peso dell'assistenza al malato. Ne consegue che "il percorso del paziente con patologia cronica deve essere pianificato nel lungo periodo e gestito in modo pro-attivo e differenziato, per rispondere ai bisogni specifici e prevenire l'insorgenza di complicanze evitabili".

ANED ha partecipato alla stesura del Piano ed è ampiamente a favore delle cure domiciliari per i nefropatici, perché considera positivo, quando possibile e a determinate condizioni, lo spostamento del baricentro delle cure verso il territorio e il domicilio del malato. Questo vale per il paziente con una malattia renale cronica, in cosiddetta terapia 
conservativa, come anche per quello che arriva alla necessità di una terapia dialitica cronica.

I trattamenti dialitici domiciliari, sempre che correttamente indicati, costituiscono una forma ottimale di terapia perché, a parità di efficacia depurativa, consentono una migliore riabilitazione del paziente, una migliore integrazione nel contesto socio-culturale in cui vive e la possibilità di mantenere un'attività lavorativa e sociale, consentendo, inoltre, di liberare risorse in termini di posti letto, personale sanitario e attività di supporto (trasporto pazienti verso i centri dialisi, logistica ambientale, stoccaggio materiali), intervenendo, così, su criticità particolarmente rilevanti, senza un impoverimento dell'offerta, ma migliorando l'assistenza sanitaria globale.

Attualmente le dialisi domiciliari, sia peritoneale che emodialisi, utilizzano sistemi tecnologici sicuri di alta qualità, supportati nei centri più avanzati dalla telemedicina, e consentono alle persone affette da IRC di eseguire la terapia sostitutiva in sicurezza, al proprio domicilio (2). Laddove i programmi di educazione e supporto assistenziale consentano al paziente e alla famiglia di superare paure e barriere si ottengono ottimi risultati clinici, con la garanzia di una buona qualità della vita.

La terapia domiciliare è più "versatile" di quella ospedaliera, in quanto consente di variare la durata di ogni singolo trattamento, l'orario nell'arco della giornata e la frequenza settimanale, a seconda delle esigenze cliniche e psico-sociali della persona.

Inoltre, il trattamento domiciliare incide in misura minore sull'attività lavorativa o sulle attività di svago, come le vacanze. Infatti, accade spesso che taluni pazienti in emodialisi, anche giovani, lascino il lavoro perché non compatibile con l'organizzazione dei centri dialisi o rinuncino a recarsi in vacanza per paura di non trovare centri disponibili o per timori legati alla gestione dell'accesso vascolare.

Altro aspetto non trascurabile è di tipo relazionale e motivazionale. Nei pazienti in dialisi presso la struttura ospedaliera più facilmente si sviluppa una dipendenza passiva dagli operatori sanitari, mentre la dialisi domiciliare incoraggia l'indipendenza e l'autonomia del malato, lo responsabilizza e accresce la fiducia in sé e verso le persone che lo assistono.

Rispetto alla dialisi domiciliare, a fronte di innegabili vantaggi, è bene che gli attori del sistema sanitario tengano a mente alcune criticità, da affrontare perché la deospedalizzazione delle cure sia effettivamente positiva per i pazienti.

È noto, infatti, che la dialisi peritoneale non possa essere eseguita da tutti i pazienti che soffrono di insufficienza renale, perché sono necessarie una certa abilità manuale nonché la capacità di prendersi cura di sé a domicilio (3). A meno che una persona non si prenda cura del paziente e non gestisca le manovre di attacco e stacco, nonché la sorveglianza della procedura.

Uno dei principali svantaggi di questa metodica è un'efficienza ridotta e, quindi, deve essere eseguita ogni giorno, e questo, alla lunga, può diventare stressante per il paziente.
Alcuni pazienti possono, poi, accettare con difficoltà la presenza di un sottile tubo (catetere) posizionato chirurgicamente e lasciato in modo permanente a livello addominale (pancia), anche se, di norma, è nascosto sotto i vestiti.

Ancora più complesso il quadro dell'emodialisi domiciliare (HHD): dagli anni 2000, l'interesse intorno a questo approccio è in crescita grazie allo sviluppo di apparecchiature sempre più "maneggevoli", ai risultati di numerosi studi che ne attestano i benefici clinici, psico-sociali ed economici, ma anche a un cambiamento culturale che vede il malato non più come soggetto passivo, ma come persona che partecipa attivamente al processo di cura (4). È un paziente sempre più informato, che acquista competenze che riguardano la gestione della propria terapia e che diventa quello che oggi definiamo "paziente esperto" e motivato.

I pazienti poco informati, viceversa, non hanno interesse a intraprendere un percorso domiciliare in cui non si sentono sicuri, hanno paura del cambiamento, si sentono sfiduciati e hanno la tendenza a delegare la terapia in una fase di fortissima depressione a causa di una condizione definitiva, cronica, da cui non guariscono.

Hanno paura di sovraccaricare la famiglia di un ulteriore peso oltre a quello della malattia stessa.

Inoltre, sono spaventati dalla gestione dell'accesso vascolare, dalla puntura della fistola, dal rischio di sanguinamento e dalla gestione dei cateteri endo-vascolari. Pazienti e familiari possono aver paura di approcciarsi con l'apparecchiatura, che sembra complessa e difficile da imparare.

Il rischio di burnout del partner/caregiver è reale e richiede una forte integrazione tra lo staff nefrologico e i servizi territoriali per dare sostegno alla famiglia.

Il periodo di addestramento è piuttosto lungo per l'HHD e deve essere effettuato da infermieri esperti dedicati e, in molti centri nefrologici, gli infermieri sono in forte carenza di organico rispetto al numero dei pazienti in dialisi e con poco tempo da dedicare all'addestramento del paziente e del caregiver.

La maggior parte delle complicanze cliniche dell'HHD sembra riconducibile alla gestione dell'accesso vascolare. Non è facile, per il partner assumersi la responsabilità di infiggere due aghi di grosse dimensioni nelle braccia del proprio familiare, con il rischio di non posizionare bene gli aghi o di causare ematomi o vere e proprie lacerazioni dei vasi. La tecnica di venipuntura a occhiello, buttonhole degli Autori anglosassoni, offre un minore rischio di false vie e di insuccessi di posizionamento. Tuttavia, se non ben gestita, sembra associata a un più alto tasso di infezioni.

Nella fase attuale, il rinnovato interesse per l'HHD sta portando recentemente alla crescita del numero di pazienti che eseguono la dialisi al domicilio. Tuttavia, sia in Italia che nel resto del mondo, la percentuale di dialisi domiciliare rispetto al numero totale dei pazienti in dialisi continua a essere molto bassa.

Con l'avvento di sistemi e apparecchiature sempre più semplici da gestire e da apprendere nella gestione, c'è da 
chiedersi cosa ostacoli realmente lo sviluppo della metodica domiciliare e quali siano le barriere, che, a fronte dei dimostrati vantaggi, ne ostacolano la diffusione.

Uno dei motivi principali almeno in Europa è che i pazienti in dialisi hanno un'elevata età media che spesso supera i settanta anni (5), sono, inoltre, gravati da patologie multiple e hanno problemi o di accesso vascolare o di accesso peritoneale. Spesso, poi, un paziente anziano ha un partner anziano che teme le manovre cruente e non accetta la responsabilità della gestione della dialisi. Sono aspetti che non devono essere sottovalutati, pena l'insuccesso precoce dei programmi di deospedalizzazione.

Per supplire in parte a questi problemi sono state proposte le cosiddette dialisi domiciliari assistite, vale a dire peritoneale ed emodialisi, condotte da un infermiere esperto.

Qui, però, sorgono alcuni problemi:

i) i costi del personale (che per l'HHD sono molto elevati, in quanto è richiesta, almeno per l'emodialisi, un'assistenza continuativa di un solo infermiere prima della procedura, che, di per sé, dura almeno 4 ore, durante e dopo);

ii) l'addestramento specifico del personale che si reca a domicilio;

iii) il collegamento funzionale e pratico con il centro madre.

Per la dialisi domiciliare peritoneale assistita, il costo del personale può essere ripartito sull'assistenza a più pazienti, dal momento che non è necessaria una presenza continuativa del personale infermieristico, se non nelle fasi di attacco e stacco.

Tuttavia, affinché la dialisi domiciliare assistita possa crescere e diffondersi, è necessario che tutte le condizioni di garanzia siano soddisfatte, facendo ricorso, per la gestione, alle risorse disponibili del Servizio Sanitario Nazionale.

La maggiore autonomia e la conseguente responsabilizzazione che derivano dall'autogestione della propria salute hanno un effetto terapeutico chiaro: i pazienti hanno una migliore percezione del loro stato di salute e possono ridurre la necessità di farmaci. Non da ultimo, la deospedalizzazione riduce, inoltre, la morbilità legata alla potenziale trasmissione di malattie infettive. Una recente survey della Società Italiana di Nefrologia eseguita in occasione della pandemia COVID-19 ha dimostrato come la diffusione della malattia nei pazienti in dialisi sia molto meno frequente nei pazienti in dialisi domiciliare peritoneale che nei pazienti che eseguono l'emodialisi presso i centri ospedalieri (6). La scelta del trattamento dialitico dipende sicuramente da valutazioni di carattere clinico, ma deve, allo stesso tempo, considerare gli aspetti psicologici ed emotivi del paziente e le aspettative e le necessità di vita del paziente e del suo contesto socio-familiare, puntando a una terapia sempre più personalizzata e consentendo al paziente di scegliere consapevolmente la propria terapia.
Per un'adeguata programmazione, occorre definire parametri ambientali, di qualità dell'assistenza, di dotazioni necessarie e di sostegno economico, ai fini di garantire la sicurezza del paziente e la gestione migliore e più tranquilla.

La proposta di ANED è che le Regioni definiscano i piani di assistenza domiciliare per le persone in dialisi, in modo da garantire il personale sanitario pubblico necessario per l'organizzazione di un'adeguata rete di caregiver. A tal proposito, l'occasione è rappresentata dagli obiettivi indicati nei piani di reclutamento del personale, che prevedono l'assunzione di medici e infermieri da destinare ad attività da svolgere nel territorio, e anche dalla figura dell'infermiere familiare, facente parte organicamente del Servizio Sanitario Nazionale. La funzione dell'assistenza dialitica domiciliare deve essere accompagnata dalla garanzia di adeguate scelte organizzative, tali da assicurare al paziente e ai suoi familiari un approccio olistico che non solo li garantisca sotto l'aspetto tecnico, ma che ne curi gli aspetti psicologici e li supporti in un percorso che, anche se domiciliare, è soverchio di sacrifici e rinunce.

Dal punto di vista economico, è necessario individuare forme di incentivi/rimborsi, volti a coprire le spese sostenute dai pazienti, e dai loro caregiver, nella gestione delle dialisi domiciliari (oltre alla messa in regola dell'ambiente e degli impianti interni, alla fornitura delle apparecchiature mediche e dei materiali e allo smaltimento dei rifiuti).

\section{Disclosures}

Conflict of interest: The authors declare no conflict of interest. Financial support: This research received no specific grant from any funding agency in the public, commercial, or not-for-profit sectors.

\section{Bibliografia}

1. Piano Nazionale della cronicità. Ministero della Salute 0021622-21/07/2016-DGPROGS-MDS-A - Allegato Utente 2 (A02). Online

2. Wilkie M. Home dialysis-an international perspective. NDT Plus. 2011;4(suppl 3):iii4-iii6. Medline

3. Li PK, Chow KM, Van de Luijtgaarden MW, et al. Changes in the worldwide epidemiology of peritoneal dialysis. Nat Rev Nephrol. 2017;13(2):90-103. CrossRef Medline

4. Walker RC, Howard K, Morton RL. Home hemodialysis: a comprehensive review of patient-centered and economic considerations. Clinicoecon Outcomes Res. 2017;9:149-161. CrossRef Medline

5. Kramer A, Pippias M, Noordzij M, et al. The European Renal Association-European Dialysis and Transplant Association (ERA-EDTA) Registry Annual Report 2016: a summary. Clin Kidney J. 2019;12(5):702-720. CrossRef Medline

6. Quintaliani G, Reboldi G, Di Napoli A, et al; Italian Society of Nephrology COVID-19 Research Group. Exposure to novel coronavirus in patients on renal replacement therapy during the exponential phase of COVID-19 pandemic: survey of the Italian Society of Nephrology. J Nephrol. 2020;33(4):725-736. CrossRef Medline 